Meta

Journal des traducteurs

Translators' Journal

\title{
La retraduction de Huckleberry Finn : Huck a-t-il (enfin) trouvé sa voix ?
}

\section{Corinne Wecksteen}

Volume 56, numéro 3, septembre 2011

URI : https://id.erudit.org/iderudit/1008328ar

DOI : https://doi.org/10.7202/1008328ar

Aller au sommaire du numéro

Éditeur(s)

Les Presses de l’Université de Montréal

ISSN

0026-0452 (imprimé)

1492-1421 (numérique)

Découvrir la revue

Citer cet article

Wecksteen, C. (2011). La retraduction de Huckleberry Finn : Huck a-t-il (enfin) trouvé sa voix ? Meta, 56(3), 468-492. https://doi.org/10.7202/1008328ar
Résumé de l'article

Les différentes traductions françaises de Adventures of Huckleberry Finn de Mark Twain ont souvent fait l'objet de critiques, qui mettaient surtout l'accent sur les lacunes traductives concernant le traitement des spécificités langagières du roman. Dans cet article, nous proposons de voir en quoi la nouvelle traduction de Bernard Hoepffner, publiée aux éditions Tristram en 2008, se démarque de certaines versions antérieures quant à la façon dont a été traduite la voix de Huck. Nous portons notre attention sur le projet de retraduction et examinons quelques aspects quantitatifs mais surtout qualitatifs du texte, avec l'étude de différents marqueurs (lexicaux, phonographologiques, morphologiques, syntaxiques et grammaticaux), qui montrent l'inventivité dont a fait preuve le traducteur. Enfin, nous nous intéressons à la réception de cette traduction en France et tentons de voir si les commentaires dithyrambiques sont justifiés ou sont susceptibles d'être nuancés.
Ce document est protégé par la loi sur le droit d'auteur. L'utilisation des services d'Érudit (y compris la reproduction) est assujettie à sa politique d'utilisation que vous pouvez consulter en ligne.

https://apropos.erudit.org/fr/usagers/politique-dutilisation/ 


\title{
La retraduction de Huckleberry Finn : Huck a-t-il (enfin) trouvé sa voix?
}

\author{
CORINNE WECKSTEEN \\ Université d'Artois, Arras, France* \\ corinne.wecksteen@univ-artois.fr
}

\begin{abstract}
RÉSUMÉ
Les différentes traductions françaises de Adventures of Huckleberry Finn de Mark Twain ont souvent fait l'objet de critiques, qui mettaient surtout l'accent sur les lacunes traductives concernant le traitement des spécificités langagières du roman. Dans cet article, nous proposons de voir en quoi la nouvelle traduction de Bernard Hœpffner, publiée aux éditions Tristram en 2008, se démarque de certaines versions antérieures quant à la façon dont a été traduite la voix de Huck. Nous portons notre attention sur le projet de retraduction et examinons quelques aspects quantitatifs mais surtout qualitatifs du texte, avec l'étude de différents marqueurs (lexicaux, phonographologiques, morphologiques, syntaxiques et grammaticaux), qui montrent l'inventivité dont a fait preuve le traducteur. Enfin, nous nous intéressons à la réception de cette traduction en France et tentons de voir si les commentaires dithyrambiques sont justifiés ou sont susceptibles d'être nuancés.
\end{abstract}

\begin{abstract}
The various French translations of Mark Twain's Adventures of Huckleberry Finn have often been criticised for their translational flaws, especially in the way they have handled the language specificities in the novel. The aim of this article is to see how the new translation by Bernard Hœpffner, published by Tristram in 2008, breaks with some earlier versions as to the way Huck's voice has been translated. Attention will be devoted to the project underlying the new translation, which will be put to the test through the examination of quantitative but mostly qualitative aspects and the study of various markers (lexical, phono-graphological, morphological, syntactic and grammatical) that show how inventive the translator has been. The last part of this article will focus on the reception of this translation in France and on the qualifications that might be brought to bear on this unanimously acclaimed work.
\end{abstract}

\section{MOTS-CLÉS/KEYWORDS}

Huckleberry Finn, inventivité, Mark Twain, retraduction, voix

Huckleberry Finn, inventiveness, Mark Twain, retranslation, voice

\section{Introduction}

Dans la conclusion de sa thèse de doctorat intitulée La traduction de la rhétorique enfantine chez Mark Twain, Jenn (2004a: 426) indique que «l'espace de jeu d'une retraduction des romans [Tom Sawyer et Huckleberry Finn] semble bien exister». Il ajoute:

Une retraduction est [...] particulièrement souhaitable pour ce dernier roman [Huckleberry Finn]. [...] [U]ne nouvelle version pourrait prendre le parti de restituer certains aspects négligés du texte. Le respect de la polyphonie, dont les implications sociales sont évidentes, constitue en soi un projet ne nécessitant nullement le placage d'une idéologie sur le texte mais participe d'une réactivation, par la traduction, de la charge 
idéologique inhérente à celui-ci. Sans dévoyer ou moderniser le roman, un certain nombre de mesures simples à mettre en place ainsi que la reconnaissance de l'importance des voix enfantines peuvent permettre d'œuvrer dans ce sens. (Jenn 2004a: 426-427)

Nous nous proposons ici de nous intéresser à la nouvelle traduction du roman de Mark Twain, Adventures of Huckleberry Finn $\left(1884 / 1977^{1}\right)$, sortie en septembre 2008 en France aux éditions Tristram ${ }^{2}$. Elle est présentée en page de couverture comme une «nouvelle traduction intégrale de Bernard Hœpffner». L'éditeur précise sur la quatrième de couverture, destinée comme on le sait à accrocher le lecteur potentiel:

Le plus souvent disponible dans des adaptations tronquées, indifférentes à sa qualité d'œuvre littéraire, Huckleberry Finn n'avait pas encore bénéficié en français d'une traduction qui rende justice à la saveur et à l'énergie incomparables du texte original.

La version intégrale - augmentée de deux longs passages inédits - qu'en offre aujourd'hui Bernard Hœpffner procurera à toutes les générations de lecteurs le sentiment d'un livre neuf, jamais lu sous cette forme.

Nous allons donc essayer d'établir en quoi cette version se démarque d'autres traductions, sur le plan quantitatif d'une part mais surtout sur le plan qualitatif. Pour faire ressortir le caractère novateur de la traduction de Hœpffner, la comparaison avec les versions de Suzanne Nétillard (publiée en 1948 et rééditée en 1993³) et d'André Bay (publiée en 1960, rééditée en $1990^{4}$ et en $1994^{5}$ ) pourra s'avérer utile. Nous laisserons le plus souvent de côté la traduction datée de William-L. Hughes, la première publiée $\left(1886^{6}\right)$, car elle s'adresse à un lectorat d'enfants et est perçue en général comme une adaptation, tandis que les deux traductions ont été considérées comme s'adressant à un public d'adultes (Lavoie 2002b: 149; Gouanvic 2004: 155), même si la version de Nétillard a ensuite été reléguée dans les collections jeunesse (Jenn 2004a: 38-39). Dans le cadre de cet article, nous essaierons de voir à l'aide d'exemples variés comment a été traitée la voix de Huckleberry Finn, qui est un des éléments essentiels de l'œuvre, laissant pour d'autres articles l'étude du parler noir de l'esclave Jim ainsi que d'autres aspects de cette nouvelle traduction.

Notre première partie servira de préambule, et nous verrons les raisons pour lesquelles une nouvelle traduction était souhaitable, ainsi que la philosophie générale du projet de Hœpffner et des éditions Tristram. Notre deuxième partie nous plongera dans le corpus, avec une étude des aspects quantitatifs, mais surtout qualitatifs de cette retraduction, notamment en ce qui a trait aux marqueurs lexicaux, phonographologiques (Demanuelli 1993: 89), morphologiques, syntaxiques et grammaticaux. Nous nous appuierons également sur les divers commentaires et remarques que le traducteur lui-même a pu faire sur ses choix de traduction, à l'aide des interviews qu'il a accordées, des réponses qu'il a pu donner aux questions qui lui ont été posées, afin de déterminer quel est son projet de traduction ${ }^{7}$ et de voir si l'annonce de l'éditeur est concrétisée dans les choix de traduction actualisés dans le texte. Enfin, dans une troisième et dernière partie, nous nous intéresserons à la réception qui a été faite de cette traduction, l'«écho critique» faisant partie des "différentes instances de consécration» du produit culturel qu'est la traduction (Gouanvic 1999: 17). 


\section{1. État des lieux : les traductions de Adventures of Huckleberry Finn de Mark Twain ou la nécessité d'une nouvelle traduction}

\subsection{La langue de Twain et les lacunes traductives}

Tous les chercheurs ${ }^{8}$ et commentateurs qui se sont intéressés aux traductions de Adventures of Huckleberry Finn n'ont pas manqué de faire le constat d'un manque:

Le lecteur français ne dispose à l'heure actuelle d'aucune traduction qui lui permette de comprendre la nature de la véritable révolution qu’a opérée ce roman dans la littérature américaine. (Maniez 1998: 82)

Or, cette révolution est perceptible dans la langue qu'utilise Twain, et qui caractérise l'œuvre littéraire qu'est Huckleberry Finn:

«Mark Twain, écrit Mr de Voto, a exercé plus d'influence sur la prose américaine que n'importe quel autre écrivain.» Il a, en effet, transformé le dialecte de la vallée du Mississippi en une langue littéraire qui, peu à peu, a presque complètement remplacé l'anglais d'Angleterre dans la prose américaine. Ses répétitions, ses gaucheries, son arrangement des mots, des accents et des pauses, ont été imités et reproduits à profusion. (Brodin 1948/1990: XXXIII ${ }^{9}$ )

La spécificité du roman réside surtout dans la place qui est donnée aux voix, et en particulier à celle de l'adolescent qu'est Huckleberry:

La langue de Huckleberry Finn est entièrement dépendante de celui qui est censé écrire le roman et de son mode de perception. Il s'agit d'un petit garçon sans éducation, jeune comme les États-Unis, dont la voix familière, gouailleuse, est capable de lyrisme lorsqu'il s'agit d'évoquer les scènes du sublime américain. C'est une innovation extraordinaire, venue du refus de Twain d'obéir à la «genteel tradition». Depuis ses débuts d'écrivain, Twain détestait la langue des lettrés, dont il trouvait l'élégance et le raffinement creux et ridicule; il voulait imaginer autre chose. Il pensait à deux modèles idéaux: la langue des enfants qu'il imaginait «intéressante», «simple», «naturelle» parce que dépourvue d'artifices, du désir de «faire des effets», et la langue de la Frontière. (Grimal 1994: 28-2910)

Naturellement, malgré le paragraphe liminaire intitulé Explanatory, dans lequel Twain donne des indications sur les dialectes utilisés dans l'œuvre, la transcription des dialectes n'est jamais rigoureusement exacte et il s'agit bien plus d'une représentation littéraire d'une réalité linguistique, qui permet de donner l'illusion de la réalité.

Ce qui fait la caractéristique de Twain, c'est cette représentation du vernaculaire, couplée à une inventivité tant linguistique que prosodique et stylistique:

Twain procède de deux manières pour arriver à cet effet vernaculaire et lyrique si particulier au livre. D’abord, il conserve à son style des caractéristiques familières (orthographiques, lexicales, syntaxiques), mais de manière non systématique pour ne pas mécaniser la langue. S'il imite le vocabulaire, choisit des monosyllabes, use de la parataxe, il travaille en même temps le rythme et tous les systèmes de variantes et de répétitions, il choisit aussi le mot rare ou inventé, le régionalisme, il étend l'utilisation des tropes bien au-delà de ce qu'une simple esthétique réaliste aurait exigé. (Grimal 1994: 29-30)

L'aspect novateur de l'écriture twainienne a fait dire à T. S. Eliot que Twain faisait partie des rares écrivains qui avaient modernisé la langue et qui avaient donné un sens plus pur aux mots de la tribu, ce qui évoque bien entendu Mallarmé: 
[T. S. Eliot] considered Twain - 'at least in Huckleberry Finn' - 'one of those writers of whom there are not a great many in any literature, who have discovered a new way of writing, valid not only for themselves but for others', and placed him with Dryden and Swift 'as one of those rare writers who have brought their language up to date, and, in so doing, "purified the dialect of the tribe". (Coveney 1985: 39; nous soulignons ${ }^{11}$ )

Tous ces éléments, qui montrent bien la spécificité de la langue de Twain, font que les traductions de Huckleberry Finn ont jusqu'ici souvent donné l'impression d'être incapables de véhiculer la même charge esthétique, privant ainsi le lecteur français d'un accès à cette nouvelle forme de littérature:

L’ampleur des problèmes évoqués par Huckleberry Finn a fait du livre un classique. Mais l'originalité de sa langue, moins perceptible de nos jours surtout en traduction, suffirait presque à expliquer la place du roman dans les lettres américaines. (Grimal 1994: 28; nous soulignons)

Il suffit d'examiner les commentaires qui ont été faits des trois traductions choisies ici comme points de comparaison avec celle de Hœpffner. Pour ce qui est de la traduction de Hughes (1886), les ajouts, omissions et autres altérations qu'il fait subir au texte font que l'on ne peut considérer sa version de Adventures of Huckleberry Finn comme une véritable traduction, l'article de Lavoie (2002a) qui y est consacré s'intitulant d'ailleurs «Traduire pour aseptiser»:

Qu'une telle production ait pu porter le nom de traduction a d'ailleurs de quoi surprendre le lecteur d'aujourd'hui: malgré la mention portée sur la page de titre, «Les aventures de Huck Finn, l'ami de Tom Sawyer, traduit avec l'autorisation de l'auteur par William-L. Hugues", il ne s'agit en fait que d'une adaptation, puisque Hugues réduit considérablement la taille de l'ouvrage (on passe de 43 chapitres à 34), et en modifie même l'intrigue [...]. Quant à la voix narrative de Huck, elle disparaît entièrement derrière une diction de jeune homme de bonne famille qui manie à la perfection le passé simple et les imparfaits du subjonctif. (Maniez 1998: 74)

Quant à la traduction de Nétillard, même si elle n'est pas parfaite, elle recueille des commentaires positifs:

Extrêmement sensible à la matérialité du signe (qu'il s'agisse de sociolectes ou de répétitions), Nétillard propose une traduction qui parvient à véhiculer un projet esthéticoidéologique très proche de celui qui fut dégagé du texte original. (Lavoie 2002b: 189)

Nétillard est un peu plus proche [que Bay] de l'esprit de Twain. Elle apparaît plus sensible au registre de Huck. [...] Mais ce sont des essais timides de rendre la parole de Huck, et son style. (Gouanvic 2004: 162)

The original translation (1886) being so far from the original, it can be considered that the 1948 version by Suzanne Nétillard is the first and by all standards the best of all those that were ever published in French and in France. (Jenn 2004b)

Enfin, comme l'indique Gouanvic (2004: 158), la traduction de Bay, qui date de 1960, ne pouvait pas rendre compte des particularités du texte de Twain puisque, dans sa "note sur la traduction» liminaire qui reprend en partie l'Explanatory de Twain, Bay rejette d'emblée l'idée de contrevenir aux règles de la langue française:

Mark Twain annonce dans une note liminaire qu'il a employé divers dialectes dans ce livre, au moins quatre, celui des nègres du Missouri, celui du Sud-Ouest, etc., sans compter les subdivisions dialectales et il nous avertit aimablement de ce fait parce qu'on 
pourrait s'imaginer que tous ses personnages s'efforcent de parler de la même façon sans tout à fait y parvenir.

En employant systématiquement le style parlé, Mark Twain, en autodidacte conscient de ses limites, a opéré une véritable révolution dans la littérature américaine, il lui a permis d'être enfin elle-même et non plus une branche de la littérature anglaise. Dans la présente traduction, nous nous sommes efforcés [sic] de conserver au récit son naturel un peu débraillé, familier, sans aller jusqu'à l'argot et en respectant autant que possible les règles de la grammaire (Bay 1960/1990: 178; nous soulignons ${ }^{12}$ )

À propos du sociolecte de Huck, Gouanvic indique que la version de Bay est bien timide par rapport à l'original:

Tout ce qu'André Bay se permet à l'occasion, c'est l'élision de la première partie de la négation, ne, dans les constructions verbales négatives [...] pour mimer le langage parlé de Huck. Il utilise aussi, parfois, des expressions familières [...]. Mais l'ensemble est un style de bon ton, bien loin du style de Huck. (Gouanvic 2004: 161)

On peut donc noter que, si progression il y a, d'une retraduction à l'autre, cette progression n'est pas forcément linéaire, puisque c'est la traduction de Nétillard qui semble recueillir le plus de suffrages, tandis que celle de Bay, qui lui est pourtant postérieure, paraît à bien des égards rendre moins bien compte de la spécificité du texte de Twain, même si c'est celle qui s'est imposée dans le paysage éditorial français (Jenn 2004a: 431).

Puisque la langue et le style de Twain semblent avoir particulièrement souffert en traduction, on comprend pourquoi les chercheurs ont appelé une nouvelle traduction de leurs vœux. Si Lavoie suggère d'utiliser un français créolisé comme option de traduction du Black English de l'esclave Jim, il reste à trouver une façon de rendre le sociolecte de Huck:

Mais encore, qu'en est-il de la langue poétique et sociolectale de Huck? Aucun traducteur n'a véritablement osé, à ce jour, faire parler Huck comme le petit garçon peu instruit qu'il est en anglais. Un projet possible serait donc celui-ci: donner à lire, en traduction, toutes les voix du roman original, c'est-à-dire, entre autres, une narration truffée de redites et de fautes linguistiques, ainsi qu'une parole noire française. Une telle entreprise de traduction permettrait dès lors de faire renaître l'œuvre de Twain et de réactiver à sa pleine mesure la charge profondément revendicatrice qu'elle véhicule. (Lavoie 2002b : 213)

La polyphonie de l'œuvre doit être au centre des préoccupations d'une retraduction, ce qui implique, entre autres, de prendre en compte ce que Jenn (2004a) appelle la "rhétorique enfantine», qui renvoie à la représentation littéraire d'un sociolecte générationnel et comporte des traits aussi bien lexicaux que syntaxiques (répétitions), ainsi que l'emploi de figures comme la litote, la comparaison ou l'hyperbole. Nous verrons plus loin si les suggestions de Lavoie («narration truffée de redites et de fautes linguistiques») ont été mises en œuvre par Hœpffner.

\subsection{Motivations et philosophie générale du projet de (re)traduction}

Ce qui a motivé cette retraduction et suscité l'intérêt de l'éditeur pour le projet, c'est bien sûr l'ensemble des carences que nous venons de signaler, tant sur le plan narratif, stylistique, esthétique ou idéologique, tous ces aspects étant imbriqués les uns dans les autres. Il n'est pas étonnant de constater que les critiques de Hœpffner sur 
les précédentes traductions portent sur leur vieillissement (les traductions anciennes sont forcément caduques) et sur leur manque de fidélité à l'esprit et à l'esthétique du texte. Ainsi, Nathalie Crom (2008), qui relate son entrevue avec le traducteur, rapporte:

[...] on n'avait tout simplement pas les moyens de lire ce livre [Les Aventures de Huckleberry Finn], faute d'une version française fidèle à l'esprit et à l'esthétique de l'original, estime le traducteur Bernard Hœpffner, à qui l'on doit la nouvelle, superbe, édition du diptyque romanesque de l'écrivain américain, qui paraît aujourd'hui chez Tristram. "J'ai bien conscience qu'il est très facile, trop facile même, de critiquer les traductions anciennes, forcément caduques. Mais dans le cas des Aventures de Huckleberry Finn, on ne peut pas faire autrement.» [...] "[j]e suis naturellement allé voir ce qui existait en français. Là, je me suis rendu compte que la dizaine de traductions existantes ne tenaient pas. »

[...] [L]e reproche majeur que Hœpffner adresse aux traductions françaises antérieures des Aventures de Huckleberry Finn, c'est évidemment de ne pas avoir voulu, ou ne pas avoir su, prendre en compte la démarche formelle de Mark Twain, sa nouveauté radicale: «[...] Twain a choisi cette fois de faire parler Huck Finn à la première personne, de faire entendre la voix d'un gamin du Sud illettré et voyou, la parole aussi des esclaves noirs, à travers le personnage de Jim. Ainsi, Twain a introduit le langage parlé dans la littérature anglo-saxonne.»(Crom 2008 ${ }^{13}$ )

Comme l'explique encore Hœpffner dans une entrevue avec Sabine Audrerie, la voix de Huck a subi des déformations telles que l'on n'entend absolument pas la parole inventée par Twain:

Bernard Hopffner: [...] Alors que Huck, qui écrit le livre à la première personne est un garçon illettré, gouailleur et s'exprime dans un langage foisonnant, on lui faisait employer un français châtié, avec imparfaits du subjonctif et passés simples. L'envie de retraduire les deux livres [Les Aventures de Tom Sawyer et Aventures d'Huckleberry Finn] s'est concrétisée grâce aux Éditions Tristram, qui nourrissaient le même projet. Ils m’ont donné une totale liberté, celle d'écrire «mal bien». (Audrerie 2008 ${ }^{14}$ )

Ainsi le projet de traduction a-t-il consisté à tenir compte de la transgression du code littéraire que constituait l'œuvre, de la matérialité des signifiants et de ces nombreuses déformations et déviations qui émaillent le texte.

Pour mener à bien ce projet, Hopffner estime que le traducteur ne doit pas s'effacer derrière le texte de départ mais au contraire adopter une attitude créative. Ainsi, à la remarque "Vous défendez la liberté du traducteur face au texte d'origine, la possibilité de réinventer le texte» (Audrerie 2008), Hœpffner répond:

Bernard Hœpffner : [...] Je ne crois pas à l'idée selon laquelle le traducteur devrait être transparent. J'ai été artisan pendant longtemps, je restaurais des objets d'ExtrêmeOrient, et il m'arrive de dire que je suis un «manuel de la traduction». Je pense que la traduction n'est pas un travail intellectuel, mais artisanal. Tout est important, la manière dont on est installé, où l'on place le livre, etc. Pour Huck Finn je n'ai pas étudié le livre, je suis allé vite. Je suis beaucoup moins sourcilleux qu'à mes débuts.

Ce qui m'intéresse pour le premier jet, c'est la dynamique. Mark Twain a écrit le livre au fil de la plume, c'est le langage qui invente les choses, un mot qui appelle l'autre. Ce premier jet fait éclore de jolies choses, après on peut revenir en détail sur le texte et l'affiner. Ainsi on touche un peu, en effet, à la création. On peut rester sur cette limite entre traduction et écriture tant qu'on se tient à l'intérieur d'un cadre éthique qui est la fidélité absolue au style et à la langue de départ. Cela pour faire passer un univers et une culture. Lire Huck Finn, c'est peut-être comprendre pourquoi les Américains ont voté Bush, c'est comprendre une culture et son histoire. (Audrerie 2008) 
Hœpffner revendique par ailleurs de recourir à l'inventivité, en manipulant la langue, en repoussant plus loin les limites de la correction grammaticale ou syntaxique, tout en notant que les normes de la langue d'arrivée peuvent constituer des freins. À la remarque concernant ce travail sur la langue ("Vous avez traduit Ulysse de Joyce, La Boîte blonde de Toby Olson, William T. Vollmann, Martin Amis ou Sorrentino, autant d'écrivains qui travaillent sur la langue»), Hœpffner répond:

Bernard Hopffner: [...] Quand par inadvertance j'écris "gripper» au lieu de "grimper» et que je le conserve, je le défends car Twain, à un autre endroit, va inventer des verbes que je ne peux pas traduire. Il attaque la grammaire américaine: j’attaque la grammaire française. Voltaire disait: «Traduire c'est faire violence à la langue d'arrivée.» Mais on doit rester en deçà: en anglais on dit d'une faute: "It's incorrect»; en français on dit: «Ce n'est pas français». Le rapport à la correction syntaxique est plus étroit, on est donc obligé de frapper moins fort.

Trop de traducteurs pensent: voilà l'état du français, c'est dans ce cadre que je dois travailler.

Tout ce qui est écrit en français est bon! J’entends parfois dire: «Ce n’est pas beau ». Mais qu'est-ce que c'est que cette esthétique? Quand j'entends un enfant dire «Je suis arrivé en retôt», s'inspirant de « retard», je trouve cela magnifique! Et d'ailleurs je suis heureux de placer ce mot dans toutes mes traductions. (Audrerie 2008).

Ces quelques remarques permettent de cerner l'optique dans laquelle se situe le travail de retraduction de Hœpffner et il convient maintenant, d'une part, de recenser les stratégies auxquelles recourt le traducteur pour mettre en œuvre son projet et de voir, d'autre part, s'il y a une cohérence dans ses choix et si les considérations théoriques se retrouvent effectivement actualisées et matérialisées dans la traduction.

\section{La (re)traduction de Bernard Hœpffner}

Avant de nous attacher dans le détail aux choix de traduction opérés par Hœpffner, il convient très rapidement de voir dans quelle mesure cette publication des éditions Tristram peut se revendiquer comme une «version intégrale».

\subsection{Aspect quantitatif}

Hœpffner donne des précisions ${ }^{15}$, sur une page intitulée «note du traducteur» qui se situe juste après la fin du roman, avant la table des matières, sur ces deux longs passages inédits mentionnés par l'éditeur sur la quatrième de couverture, le premier d'entre eux s'étalant sur six pages:

Au chapitre 9, le passage qui commence p. 78 par «On s'est déjà trouvés dans un orage ici...», jusqu'à "...voi' les lignes et de les appâter.», p. 83, figurait sur la moitié du manuscrit des Aventures de Huckleberry Finn, retrouvée il y a seulement quelques années. Il avait sans doute été retiré du roman à la demande de l'épouse de Mark Twain. Il mérite d'être remis à sa place dans ce livre plutôt qu'en appendice. On le trouve imprimé pour la première fois dans l'édition des Adventures of Huckleberry Finn, sous la direction de Victor Fischer et Lin Salamo, University of California Press, 2003. (Hœpffner 2008 ${ }^{16}$ )

Pour ce qui est du second passage inédit, il s'agit du fameux épisode du radeau et Hœpffner indique qu'il se trouvait dans Life on the Mississippi: 
Au chapitre 16, le passage qui commence p. 135 par «Mais vous savez bien qu'une jeune personne...", jusqu'à "... j'étais sacrément content d'être de nouveau chez nous.», p. 153, éliminé par le premier éditeur, avait été repris par Mark Twain dans le chapitre 3 de La Vie sur le Mississippi. Il est replacé ici à l'endroit où il se trouvait à l'origine. (Hœpffner 2008)

Hœpffner précise que c'est l'éditeur qui a éliminé le passage, tandis que dans l'appendice de la version publiée chez Penguin, l'explication est moins tranchée et l'on ne sait pas si c'est Twain ou l'éditeur qui a décidé de ne pas inclure le passage (Twain 1884/1985: $371^{17}$ ).

Quoi qu'il en soit, l'inclusion de ces passages dans cette nouvelle édition permet de donner une vision plus complète de l'œuvre, que ce soit sur le plan littéraire, esthétique ou narratif (Jenn 2004a: 427, note 674).

\subsection{Aspects qualitatifs}

Même si Twain mentionne dans son Explanatory (Twain 1884/1977: 2) les différents dialectes représentés dans le roman, nous suivrons la terminologie proposée par Jenn dans sa thèse (2004a: 60) et qualifierons de vernaculaire la langue de Huck, ce qui ne restreint pas le propos à un espace géographique limité mais permet d'englober la dimension sociologique et la «rhétorique enfantine» (Jenn 2004a). Nous allons nous consacrer dans cette partie à l'étude de quelques caractéristiques de la traduction de Hœpffner sur le plan lexical, phonographologique, morphologique, syntaxique et grammatical.

\subsubsection{Marqueurs lexicaux}

\subsubsection{Inventivité et néologismes}

Nous avons dit plus haut que Hœpffner insistait sur la nécessité pour le traducteur d'être inventif et l'on retrouve cet aspect dans la remarque suivante, où il prône le recours au français du Xxi ${ }^{\mathrm{e}}$ siècle («le parler d'aujourd'hui»), dans ses diverses composantes, sociologiques («rue, métro») et générationnelles («les adolescents»), en incluant les néologismes utilisés par cette classe d'âge:

Bernard Hœpffner: [...] Je connaissais bien le texte, mais il me fallait trouver une technique pour rendre ce langage unique. J'ai d'abord énormément relu Queneau, et Céline dans une moindre mesure, deux écrivains qui ont réussi à introduire le parler commun dans l'œuvre littéraire, et ont opéré dans la littérature française l'équivalent de la transformation du langage lancée par Twain pour la littérature américaine - lui cinquante ans auparavant. Ensuite j'ai énormément écouté le parler d'aujourd'hui, dans la rue, dans le métro, ou même les adolescents autour de moi, leurs néologismes, dont certains figurent dans la bouche de Huck (Audrerie 2008).

On remarque tout d'abord que Hœpffner utilise des néologismes pour rendre des néologismes du texte de départ (TD), que ce soit sur le plan nominal, adjectival ou verbal, comme avec l'exemple ci-dessous où majestying, verbe formé par conversion du nom majesty, est traduit par majestiser, là où Nétillard et Bay optent pour «donner du (votre) Majesté» (un des hommes recueillis à bord du radeau prétend être le roi légitime de France): 
(1a) So Jim and me set to majestying him, and doing this and that and t'other for him [...].

(Twain 1884/1977: 102)

(1b) Alors Jim et moi on s'est mis à le majestiser, et à faire ceci, et cela [...].

(Twain 1884/2008, traduit par Hopffner: 206)

Mais Hœpffner insère également des néologismes dans le texte d'arrivée (TA), là où il n'y en avait pas dans le $\mathrm{TD}$, ce qui peut être considéré comme une façon de compenser d'autres éléments du TD non rendus par ailleurs. On retrouve ainsi l'utilisation de gripper, revendiquée plus haut (voir 1.2.) par Hœpffner :

(2a) Then we started for the house, and I went in the back door - you only have to pull a buckskin latch-string, they don't fasten the doors - but that warn't romantical enough for Tom Sawyer: no way would do him but he must climb up the lightning rod. But after he got up half-way about three times, and missed fire and fell every time, and the last time most busted his brains out, he thought he'd got to give it up; $[\ldots]$

(Twain 1884/1977: 185)

(2b) Alors on est repartis vers la maison, et je suis rentré par la porte de derrière - il suffisait de soulever l'attache en cuir de la clenche, ils verrouillent pas les portes - mais c'était pas assez romantique pour Tom Sawyer; y fallait absolument qu'il grippe par le paratonnerre. Quand il a eu grippé trois fois, qu'il a loupé son coup et qu'il est tombé chaque fois, et quand la dernière fois il s'est presque fait éclater la cervelle, il s'est dit que ça suffisait.

(Twain 1884/2008, traduit par Hopffner: 359)

Là où d'autres traducteurs ont tenté de rendre compte de l'oralité du texte grâce à la ponctualisation (Nétillard ajoute un point d'exclamation à la fin de la deuxième phrase), Hœpffner joue plus sur l'aspect lexical, avec le néologisme raisonnabilité, et syntaxique, en gardant la répétition que je dis:

(3a) "Now you're talking!" I says; "your head gets leveler and leveler all the time, Tom Sawyer," I says.

(Twain 1884/1977: 194)

(3b) - Ça, c'est parler! Ta tête se remet de plus en plus d'aplomb, Tom Sawyer!

(Twain 1884/1993, traduit par Nétillard 1948: 290)

(3c) «Ça c'est parler! que je dis; ta raisonnabilité va grandissant tout le temps, Tom Sawyer, que je dis. [...]»

(Twain 1884/2008, traduit par Hopffner: 374 )

Hœpffner justifie le recours aux néologismes et à l'inventivité par l'évolution des pratiques traductives et invoque le principe de la compensation, pour une fidélité qui doit se juger à l'aune du texte dans son ensemble et non mot pour mot:

Bernard Hœpffner. - [...] Nos manières de traduire évoluent aussi. On prend beaucoup plus de risques aujourd'hui. [...] Par exemple, comme je vis avec deux adolescents qui sont trilingues, je leur ai emprunté certains néologismes. Tenez, dans le chapitre 2, le néologisme "misérabilité», ça vient d'eux. Le mot ne figure pas chez Twain, mais je suis sûr que s'il l'avait entendu, il l'aurait mis dans la bouche de Huck. En l'employant, je suis un peu plus gonflé que lui. Mais trois mots au-dessus, je le suis plutôt moins... C'est donc pour lui être fidèle que, parfois, je suis infidèle à son texte (Leménager $\left.2008^{18}\right)$. 
Le néologisme misérabilité est ainsi utilisé pour traduire miserableness:

(4a) Next I got to itching underneath. I didn't know how I was going to set still. This miserableness went on as much as six or seven minutes; but it seemed a sight longer than that.

(Twain 1884/1977: 10)

(4b) Ensuite ça m’a démangé en dessous. Je savais plus comment j’allais rester assis sans bouger. Cette misérabilité a continué au moins six ou sept minutes; mais ça paraissait sacrément plus long.

(Twain 1884/2008, traduit par Hopffner: 17)

On remarque en outre que, comme il l'avait indiqué (voir citation en 1.2.), Hopffner a en effet placé l'expression néologique en retôt dans sa traduction:

(5a) But of course I forgot all about driving slow, on accounts of being glad and full of thinking; so I got home a heap too quick for that length of a trip.

(Twain 1884/1977: 178)

(5b) Mais naturellement, j’ai complètement oublié que je devais traîner, du fait que j'étais content et que je réfléchissais; alors je suis rentré très en retôt pour un trajet aussi long.

(Twain 1884/2008, traduit par Hopffner: 346-347)

Tout comme pour les néologismes, on constate que Hœpffner, contrairement aux trois autres traducteurs, utilise des mots-valises pour rendre certains mots-valises du TD, le terme préordestination fusionnant préordination et prédestination:

(6a) but everybody said it was a good sermon, and they all talked it over going home, and had such a powerful lot to say about faith, and good works, and free grace, and preforeordestination, and I don't know what all [...].

(Twain 1884/1977: 90)

(6b) mais tout le monde a dit que c'était un bon sermon, et ils en ont tous discuté en rentrant, et ils avaient tellement à dire sur la foi, et les bonnes œuvres, et la faveur imméritée, et la préordestination, et je ne sais pas quoi d'autre encore [...].

(Twain 1884/2008, traduit par Hopffner: 185)

mais qu'il en insère aussi en d'autres endroits, comme avec tintamaramdam (tintammarre + ramdam):

(7a) then comes a h-wack! - bum! bum! bumble-umble-um-bum-bum-bum-bum and the thunder would go rumbling and grumbling away, and quit - and then rip comes another flash and another sockdolager.

(Twain 1884/1977: 104)

(7b) et puis arrivait un ch-crac! - boum! boum! baoum-boum-oum-boum-boumboum-badaboum - et le tonnerre se mettait à gronder et à grommeler, et puis il s'arrêtait - et puis vlam, un autre éclair explose, et puis un autre tintamaramdam.

(Twain 1884/2008, traduit par Hopffner: 210)

On le constate, Hœpffner n'hésite pas à mettre en œuvre les mêmes moyens que Twain, comme les néologismes ou les mots-valises. Il semble même aller plus loin, en introduisant des termes inventés là où il n'y en avait pas dans le TD, mais ceci pourrait correspondre à une tentative de compensation à l'échelle générale du roman. 


\subsubsection{Niveaux de langue}

À côté de cette inventivité lexicale, Hopffner utilise également toute la palette des niveaux de langue, par le recours à des termes anciens:

(8a) I begun to suspicion something.

(Twain 1884/1977: 77)

(8b) J'ai commencé à suspicionner quelque chose.

(Twain 1884/2008, traduit par Hopffner: 162)

Là où l'on trouve suspicion utilisé par Huck comme verbe, alors qu'il s'agit en réalité d'un nom en anglais, Hœpffner opte pour suspicionner, qui est répertorié dans le Dictionnaire du Moyen Français (1330-1500) ${ }^{19}$ mais que l'on trouve aussi chez l'auteur antillais Raphaël Confiant, à partir du substantif suspicion (Sagols 2005).

Il utilise également des termes familiers, mouscaille étant répertorié comme populaire par le Trésor de la langue française informatisé $(T L F i)^{20}$ et comme familier et datant de 1880 par le Petit Robert ${ }^{21}$ :

(9a) "They're in an awful peck of trouble, and -".

(Twain 1884/1977: 62)

(9b) «Ils sont dans une mouscaille terrible, et...».

(Twain 1884/2008, traduit par Hopffner: 116)

Il opte pour des termes argotiques, comme attrimer ${ }^{22}$, qui veut dire prendre et qui traduit la forme de participe incorrect catched, là où Hugues ne rend pas le passage, tandis que Nétillard et Bay utilisent tous deux être pris:

(10a) Every minute it was getting earlier, now, and pretty soon some of them watchers would begin to stir, and I might get catched - catched with six thousand dollars in my hands that nobody hadn't hired me to take care of.

(Twain 1884/1977: 143)

(10b) Chaque minute maintenant, le jour se rapprochait, et bientôt un des veilleurs allait se réveiller, et on pourrait m'attrimer - m'attrimer avec six mille dollars entre les mains, et personne il m'avait demandé de m'en occuper.

(Twain 1884/2008, traduit par Hopffner: 282)

Il recourt à des termes mêlant diachronie et niveau de langue. C'est le cas de badigoinces, terme d'argot vieilli qui date du $\mathrm{XvI}^{\mathrm{e}}$ siècle mais qui était usité à l'époque de Twain (voir le TLFi):

(11a) $[\ldots]$ and Tom he turned kinder blue around the gills [...].

(Twain 1884/1977: 198)

(11b) Tom devint pâle comme la mort.

(Twain 1884/1993, traduit par Nétillard 1948: 296)

(11c) [...] et Tom devient bleu autour des ouïes [...].

(Twain 1884/1990, traduit par Bay 1960: 359)

(11d) [..] et Tom, il est devenu genre tout bleu autour des badigoinces [...].

(Twain 1884/2008, traduit par Hopffner: 382)

En revanche, le niveau de langue est plus soutenu chez Nétillard en raison de la comparaison pâle comme la mort, tandis que Bay opte pour une traduction littérale qui ne nous paraît pas naturelle. De nouveau, Hœpffner paraît être plus proche de 
l'esprit du TD que ses prédécesseurs, d'autant qu'il utilise "genre + adjectif» pour traduire kinder (variante phonographologique de kind of), structure dont Jenn (2004a: 386-389) recommande l'emploi systématique comme traduction de kind of pour connoter le parler des jeunes, son emploi étant d'ailleurs susceptible d'intégrer la rhétorique enfantine.

\subsubsection{Technolecte}

En outre, dernier point concernant le lexique, Hœpffner indique avoir prêté une attention particulière au technolecte lié au Mississippi:

Bernard Hœpffner. - [...] J'ai ainsi fait un énorme effort pour utiliser un vocabulaire fluvial, et non pas maritime comme c'était le cas, selon un article que j'ai trouvé sur le web, dans les deux dernières traductions de «Huck». Sans cet article, je ne m'en serais sans doute pas aperçu autant. J'ai mis des jours et des jours, par exemple, à trouver le mot «javeau» dans des textes sur les trains de flottage au Canada. Vous ne le connaissiez pas? Moi non plus bien sûr... Et en anglais aussi, c'est un mot que personne ne connaît. Peut-être qu'il ne sera pas plus compréhensible en français, mais au moins, un marinier ne pourra pas dire que j'ai écrit des âneries (Leménager 2008).

L'article auquel Hœpffner fait référence est vraisemblablement celui de Ronald Jenn, publié dans la Revue française d'études américaines en 2003 et intitulé «Transferring the Mississippi: Lexical, Literary and Cultural Aspects in Translations of Adventures of Huckleberry Finn ", où l'auteur indique justement que le terme technique équivalent à tow-head est javeau (Jenn 2003: 61), là où l'on trouve pas moins de huit termes différents pour rendre tow-head dans les traductions de Hughes, Nétillard et Bay: anse, promontoire, pointe de terre, presqu'île de sable, crique; île, îlot, banc de sable (Jenn 2003: 60). Voici un passage où figure ce terme:

(12a) soon as night was most gone, we stopped navigating and tied up - nearly always in the dead water under a tow-head; [...].

(Twain 1884/1977: 96)

(12b) dès que la nuit était presque finie, on cessait de naviguer et on amarrait - presque toujours dans les eaux calmes derrière un javeau; [...].

(Twain 1884/2008, traduit par Hopffner: 195)

Néanmoins, si l'on en croit Jenn, le terme javeau n'est peut-être pas le plus à même de rendre compte de toutes les strates de sens et de toutes les implications littéraires du terme technolectal:

The existence of this term in French does not make its use compulsory within the context of what is first and foremost a literary text. Because they refer to "white-blond hair" tow-heads are also part and parcel of a process that provides the River with human features. Technically an equivalent of tow-head, javeau does not carry all of these connotations (Jenn 2003: 68, note 7).

Aussi Jenn fait-il une autre proposition dans sa thèse:

Cependant, dans un projet de retraduction du roman, les connotations qui lient towhead à la fois au corps humain (les cheveux) et à l'enfance (une jeune île), pourraient être conservées en le traduisant par "têt'-blond' " une création suggérant la jeunesse et la blondeur (les tow-heads sont faits de sable) (Jenn 2004a: 370, note 617). 
On pourra bien sûr s'interroger sur le bien-fondé de cette suggestion, dans la mesure où l'attention accordée aux connotations attachées au terme se fait alors peutêtre au détriment de la compréhension, le lecteur risquant de rester perplexe devant une phrase telle que: "on amarrait - presque toujours dans les eaux calmes derrière une têt'-blond'».

\subsubsection{Marqueurs phonographologiques}

Hœpffner s'efforce de rendre compte des déformations orthographiques présentes dans le TD, contrairement aux autres traducteurs qui, dès le début du roman, procèdent à une normalisation en utilisant civiliser:

(13a) The Widow Douglas, she took me for her son, and allowed she would sivilize me; [...].

(Twain 1884/1977: 7)

(13b) La Veuve Douglas, elle m'a pris chez elle comme son fils et elle se disait qu'elle allait me siviliser; [...].

(Twain 1884/2008, traduit par Hopffner: 11)

Il en introduit également dans le TA là où ne figure aucun marqueur spécifique à cet endroit, étant entendu qu'il en figure bien sûr ailleurs dans le TD:

(14a) She flung herself at Tom, crying, and says: [...].

(Twain 1884/1977: 222)

(14b) Tante Sally s'est jetée sur Tom en pleurant, et elle s'esclame: [...].

(Twain 1884/2008, traduit par Hopffner: 425)

Toujours dans la perspective de rendre compte de l'oralité, il faut souligner les phénomènes d'écrasement, d'élision:

(15a) Buck and his ma and all of them smoked cob pipes, except the nigger woman, which was gone, and the two young women.

(Twain 1884/1977: 82)

(15b) Buck et sa maman et tous les autres fumaient des pipes de maïs, excepté la négresse, qu'était plus là, et les deux jeunes femmes.

(Twain 1884/2008, traduit par Hoeffner: 170)

Cet écrasement permet de rendre compte de l'incorrection grammaticale présente dans le TD, où which est employé au lieu de who.

La réduction phonétique, avec la forme pis au lieu de puis, est un autre moyen de rendre la langue utilisée par les enfants, comme le propose également Jenn (2004a: 352):

(16a) “[...] And wouldn't he throw style into it? - wouldn't he spread himself, nor nothing? [...]."

(Twain 1884/1977: 57)

(16b) «[...] Et pis, il y mettrait du style! - et pis, il gonflerait tout ça autant qu'il peut. $[\ldots] »$

(Twain 1884/2008, traduit par Hopffner: 107)

On relève aussi des phénomènes de crase, que Bernard Dupriez (1984: 139) définit comme la «contraction de deux syllabes en une». Dans l'extrait suivant, la locution quelque chose devient kekchose: 
(17a) "And besides," I says, "we might borrow something worth having, out of the captain's stateroom. Seegars, $I$ bet you [...].»

(Twain 1884/1977: 57)

(17b) «Et en plus, que je lui dis, on pourrait emprunter kekchose qu'en vaut la peine, dans la cabine du capitaine. Des cigares, je te parie [...].

(Twain 1884/2008, traduit par Hopffner: 107)

On aura bien sûr remarqué que dans le TD, la déformation ne portait pas sur

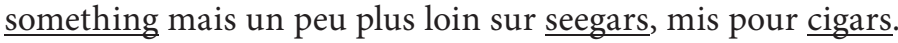

Dans l'exemple qui suit, la contraction de parce que en pasque s'accompagne d'autres types de marqueurs que nous verrons plus bas, comme l'effacement de la négation (je me suis plus intéressé), là où, dans le TD, on avait la double négation (I didn't care no more, I don't take no stock) et l'utilisation de l'adjectif à la place de l'adverbe (a considerable long time):

(18a) but by-and-by she let it out that Moses had been dead a considerable long time; so then I didn't care no more about him; because I don't take no stock in dead people.

(Twain 1884/1977: 8)

(18b) mais au bout d'un moment elle a lâché que Moïse était mort depuis vraiment très longtemps; alors je me suis plus intéressé à lui; pasque je me fiche pas mal des morts.

(Twain 1884/2008, traduit par Hopffner: 12)

On peut repérer également des amalgames syntagmatiques, qui consistent à «exprimer plusieurs syntagmes, voire plusieurs assertions, en un seul mot phonétique» (Dupriez 1984: 37):

(19a) Then I slipped down to the ground and crawled in amongst the trees, and sure enough there was Tom Sawyer waiting for me.

(Twain 1884/1977: 9)

(19b) Et puis je me suis laissé glisser jusqu'à terre et je me suis enfoncé sous les arbres, et voilatipa que Tom était là qui m’attendait.

(Twain 1884/2008, traduit par Hœpffner: 15)

Pour rendre l'oralité enfantine, Hœpffner utilise donc des figures de discours variées, qui ne sont pas sans rappeler l'écriture d'un Queneau dans Zazie dans le métro et dans les Exercices de style ou d'un Céline dans Voyage au bout de la nuit, deux auteurs dont se réclame Hœpffner et chez qui l'on retrouve des occurrences similaires ${ }^{23}$.

\subsubsection{Marqueurs morphologiques}

En ce qui concerne la morphologie, Hœpffner recourt fréquemment à la métanalyse, phénomène où «les unités de langage sont découpées et analysées autrement par celui qui entend que par le locuteur" (Dupriez 1984: 285), ce qui est typique des créations issues de l'étymologie populaire. Là où Nétillard et Bay rendaient la déformation phonographologique du TD (yaller janders pour yellow jaundice) par la jaunisse, Hœpffner utilise l'agglutination, avec l'ajaunisse:

(20a) "How's it a new kind?"

"Because it's mixed up with other things." 
"What other things?"

"Well, measles, and whooping-cough, and erysiplas, and consumption, and yaller janders, and brain fever, and I don't know what all."

(Twain 1884/1977: 153)

(20b) «Comment, c'est une nouvelle sorte?»

«Pasque c'est mélangé à d'autres choses.»

«Quelles autres choses?»

«Eh bien, la varicelle, et la coqueluche, et l'éryspelle, et la consomption et

l'ajaunisse, et la fièvre cérébrale, et je sais pas quoi d'autre.»

(Twain 1884/2008, traduit par Hopffner: 300)

Le phénomène inverse de la déglutination peut également apparaître là où aucun marqueur spécifique ne figure dans le TD à cet endroit, la forme l'a madoué correspondant au découpage erroné de l'amadouer:

(21a) Then he went to work on the nigger, coaxing him and petting him, and asking him if he'd been imagining he saw something again.

(Twain 1884/1977: 196)

(21b) Ensuite il s'est occupé de l'autre nègre, il l'a madoué et câliné, et il lui a demandé s'il avait de nouveau imaginé quelque chose.

(Twain 1884/2008, traduit par Hopffner: 379)

La stratégie adoptée par Hœpffner concerne non seulement le plan morphologique, mais aussi le plan morphosyntaxique, avec l'effacement du ne de la négation, fréquemment utilisé par de nombreux traducteurs pour rendre l'oralité, mais utilisé de manière systématique par Hœpffner au tout début du roman:

(22a) You don't know about me, without you have read a book by the name of "The Adventures of Tom Sawyer," but that ain't no matter. That book was made by Mr. Mark Twain, and he told the truth, mainly. There was things which he stretched, but mainly he told the truth. That is nothing. I never seen anybody but lied, one time or another, without it was Aunt Polly, or the widow, or maybe Mary. Aunt Polly - Tom's Aunt Polly, she is - and Mary, and the Widow Douglas, is all told about in that book - which is mostly a true book; with some stretchers, as I said before.

(Twain 1884/1977: 7)

(22b) Vous savez rien de moi si vous avez pas lu un livre qui s'appelle Les Aventures de Tom Sawyer, mais ça mange pas de pain. Ce livre, c'est Mr Mark Twain qui l'a fait, et il a dit la vérité vraie, en grande partie. Certaines choses, il les a exagérées, mais en grande partie il a dit la vérité. Ça fait rien. J'ai jamais connu quelqu'un qu'a pas menti une fois ou une autre, sauf tante Polly, ou la veuve, ou peut-être Mary. Tante Polly - elle, c'est la tante Polly de Tom - et Mary, et la veuve Douglas, on en parle dans ce livre, qui dans l'ensemble reflète la vérité; avec quelques exagérations, comme je l'ai dit tout à l'heure.

(Twain 1884/2008, traduit par Hopffner: 11)

Cet incipit est très important puisqu'il donne le ton du roman. Il a été traité différemment dans les trois autres traductions, avec aucune marque de langue relâchée dans la version de Hughes, qui procède déjà à des modifications substantielles du texte (ajouts), peu de marqueurs de ce type dans la traduction de Nétillard (si ce n'est la présence de l'interjection Bah! et du terme menteries, répertorié soit comme vieilli soit comme régionalisme), la retraduction de Bay étant plus mixte, avec un 
recours non systématique à la négation tronquée (Vous ne me connaissez pas vs J'ai jamais rencontré personne), l'utilisation de la dislocation (C'est un certain Mark Twain qui l'a écrit, ce livre), typique de l'oralité, mais aussi l'emploi du subjonctif (qui n'ait pas menti), qui n'est pas caractéristique d'une langue relâchée, ceci menant au syncrétisme, condamné par Berman (1985/1999: 38). Dans la version de Hœeffner, l'entrée en matière paraît plus incisive et plus conforme aux caractéristiques et à l'esprit du TD.

\subsubsection{Marqueurs syntaxiques et grammaticaux}

\subsubsection{Phénomènes de répétition}

Nous allons nous intéresser d'abord à un trait stylistique majeur dans le roman, qui est la récurrence de la coordination $\underline{\text { and }}$, dont l'utilisation participe ici à la mise en place d'un discours spécifique à l'enfance, qui joue sur la redondance et la répétition. Ainsi, au début du chapitre 40, la narration est-elle constituée d'un paragraphe d'une seule phrase composée d'une enfilade de propositions reliées dix-huit fois par la conjonction de coordination and:

(23a) We was feeling pretty good, after breakfast, and took my canoe and went over the river a fishing, with a lunch, and had a good time, and took a look at the raft and found her all right, and got home late to supper, and found them in such a sweat and worry they didn't know which end they was standing on, and made us go right off to bed the minute we was done supper, and wouldn't tell us what the trouble was, and never let on a word about the new letter, but didn't need to, because we knowed as much about it as anybody did, and as soon as we was half up stairs and her back was turned, we slid for the cellar cubboard and loaded up a good lunch and took it up to our room and went to bed, and got up about half-past eleven, and Tom put on Aunt Sally's dress that he stole and was going to start with the lunch, but says: [...]

(Twain 1884/1977: 212)

Nous ne nous attarderons pas sur la traduction de Hughes, qui est très condensée et ne comporte aucun et coordonnant des propositions mais un nombre important de segmentations d'une part, et d'enchâssements de propositions relatives d'autre part. Pour ce qui est de Nétillard et de Bay, Gouanvic (2004: 162) indique qu'ils procèdent à une normalisation de la syntaxe, ou «rationalisation» en langage bermanien (Berman 1985/1999: 53).

(23b) Après le déjeuner, on se sentait bien en forme, aussi on prit le canot et on partit à la pêche avec notre déjeuner dans notre poche; on s'amusa bien, on jeta un coup d'œil au radeau, qui n'avait pas eu de mal, et on rentra tard à la maison pour le souper.

On les trouva tous tellement inquiets et tracassés qu'ils ne savaient plus s'ils se tenaient sur les pieds ou sur la tête, et ils nous envoyèrent au lit dès la dernière bouchée avalée, sans vouloir nous dire ce qui se passait et sans souffler mot de la lettre; mais ça n'était pas la peine, puisqu'on était mieux renseignés là-dessus que n'importe qui. Dès qu'on fut dans les escaliers et qu'ils eurent le dos tourné, on se glissa jusqu'au placard de la cave pour y prendre un bon casse-croûte qu'on monta dans notre chambre, et puis on se coucha.

On se releva vers onze heures et demie, et Tom enfila la robe de tante Sally, qu'il avait volée; on allait se mettre à casser la croûte quand il dit tout d'un coup : [...].

(Twain 1884/1993, traduit par Nétillard 1948: 316) 
(23c) Après le petit-déjeuner, Tom et moi, nous nous sentions joliment frais et dispos. Nous avons pris mon canoë, des provisions, et nous sommes allés pêcher dans la rivière. On est aussi allés voir le radeau; bref, une bonne journée. Quand on est revenus à l'heure du dîner, la famille était dans tous ses états; dès qu'on s'est levés de table ils nous ont envoyés coucher; impossible de leur faire dire ce qu'ils avaient. De la lettre, pas un mot. Mais nous, on savait à quoi s'en tenir. Tante Sally avait pas plus tôt tourné le dos qu’on s'est glissés jusqu’au garde-manger de la cave; là, on a fait des provisions qu'on a emportées dans notre chambre. Puis on s'est couchés. A onze heures et demie, on était de nouveau debout. Tom a enfilé la robe qu'il avait chipée à tante Sally; il allait se mettre à manger quand il a demandé: [...].

(Twain 1884/1990, traduit par Bay 1960: 371)

La disposition change dans la traduction de Nétillard: là où l'on n'avait qu'un seul paragraphe en anglais, on en trouve trois en français. Par ailleurs, le recours fréquent, dans ces deux traductions, au point et au point-virgule, marque de ponctuation dont le statut n'est certes pas toujours aisé à cerner, fait perdre au texte son enchaînement de propositions en cascade et sa spécificité rythmique et fait basculer le texte dans la parataxe. Il n'y a plus que sept et dans la traduction de Nétillard, qui compte sept phrases (séparées par un point ou un point-virgule). En outre, la traductrice introduit en parallèle une forme de hiérarchisation entre les propositions: utilisation de aussi, conjonction marquant la relation de cause à conséquence, recours à une proposition infinitive (pour y prendre un bon casse-croûte), à des propositions subordonnées conjonctives (sans vouloir nous dire...) et relatives (radeau, qui n'avait pas eu de mal..., casse-croûte qu'on monta...), éléments qui font verser le texte dans l'hypotaxe. Le TA oscille donc entre la parataxe et l'hypotaxe, ce qui ne rend pas l'organisation syntaxique du TD. Pour ce qui est de Bay, on ne relève qu'un seul et et la présence de quinze phrases (séparées par un point ou par un point-virgule). On peut remarquer l'utilisation d'énoncés sans verbe (bref, une bonne journée; impossible de leur faire dire ce qu'ils avaient. De la lettre, pas un mot), qui confèrent certes un style oral à l'extrait, mais qui, au contraire de l'original, nous semblent donner l'impression d'un style plutôt incisif et saccadé en lieu et place d'une narration qui procède par accumulation, en déroulant le fil chronologique de l'action.

Voyons ce qu'il en est de la dernière traduction:

(23d) On se sentait plutôt en forme, après le petit-déjeuner, alors on a pris mon canoë et on est partis sur le fleuve avec notre dîner, pour pêcher, et on s'est bien amusés; on a été jeter un coup d'œil sur le radeau et on l'a trouvé en bon état, et puis on est arrivés en retard pour le souper; on les a tous trouvés tellement inquiets qu'ils savaient plus s'ils étaient debout ou couchés, et ils nous ont envoyés directement au lit une fois le souper terminé, sans vouloir nous dire ce qui se passait, ni nous dire quoi que ce soit sur la nouvelle lettre, ce qui faisait rien, puisqu'on la connaissait tout aussi bien que n'importe qui et, dès qu’on est arrivés au milieu de l'escalier et que tante Sally a eu le dos tourné, on s'est glissés à la cave et on a pris un bon casse-croûte dans le placard avant de remonter se coucher; on s'est relevés vers onze heures et demie, Tom a enfilé la robe de tante Sally qu'il avait volée et allait s'enfiler le casse-croûte, mais il demande: [...].

(Twain 1884/2008, traduit par Hopffner: 407) 
C'est chez Hœpffner que l'on retrouve le plus de et, au nombre de huit, accompagnés d'un et puis, qui renforce la notion de successivité; même si l'on note également l'utilisation d'adverbes (alors, exprimant la conséquence), de virgules, de points-virgules, de propositions conjonctives (sans vouloir nous dire...), c'est dans cette traduction que le nombre de phrases est le moins élevé (quatre) et se rapproche le plus de l'original. En outre, le traducteur a donné lui-même des indications sur le nombre global de et figurant dans sa traduction et a justifié cet usage, même s'il s'agit de «faire violence» au français:

Bernard Hopffner. - [...] Tenez, je me suis aperçu en relisant ma traduction de «Huck» qu'il y avait énormément de «et», ce qui est une erreur classique de débutant, car la langue anglaise utilise beaucoup plus de «and» que le français de «et » (à cause de la Bible en particulier). Je ne comprenais pas. J'ai fait un calcul, ça a été facile avec l'ordinateur: je me suis trouvé avec presque $6 \%$ de «and» dans «Huck Finn». J'ai fait le calcul sur trente autres livres, c'est un maximum! Aucun n'atteint cette proportion - sauf la Bible de King James, qui atteint 6,3 parce que toutes les phrases commencent par «And». Dans ma traduction, les 6\% de «and» deviennent 4,75\% de «et». C'est évidemment bien plus que dans tout autre livre français. Dans la première traduction du roman, il y en avait $2 \%$. On peut me dire que ce n'est pas très français, mais je m'en fous: ce que je veux, c'est «faire violence» au français. Pourquoi cet usage du «et» ne pourrait-il pas entrer dans la langue française après tout? J'ai ce fantasme hérité des traducteurs de la Renaissance, qui ont changé les langues... (Leménager 2008).

Jenn quant à lui pense que la traduction de chaque and par et est impossible car elle alourdirait considérablement le texte, et il propose d'utiliser pis, forme orale et atténuée de puis, qui «semble plus à même de supporter la réitération et signale immédiatement le caractère oral du discours [...] et possède, de plus, des connotations enfantines certaines»(Jenn 2004a: 352).

Toujours sur le plan de la répétition, on peut remarquer que Hopffner, contrairement à Nétillard et à Bay, a préservé telles quelles les répétitions de phrases ou de syntagmes présentes dans le TD:

(24a) It made me so sick I most fell out of the tree. I ain't agoing to tell all that happened - it would make me sick again if I was to do that.

(Twain 1884/1977: 94)

(24b) Ça m'a rendu tellement malade que j'ai failli tomber de l'arbre. Je m'en vais pas raconter tout ce qui s'est passé - ça me rendrait de nouveau malade de la faire.

(Twain 1884/2008, traduit par Hopffner: 192)

Ces exemples (et d'autres que nous ne citons pas) tendraient à montrer que Hopffner a tenu compte de la valeur stylistique de and, la répétition de cette coordination dans le discours de Huck participant de ce que Jenn (2004a) appelle la «rhétorique enfantine», et qu'il n'hésite pas à aller à l'encontre de la pratique répandue qui consiste à supprimer les répétitions en les remplaçant par des synonymes (Ben-Ari 1998).

\subsubsection{Constructions syntaxiques}

Sur le plan syntaxique, Hœpffner recourt à des constructions typiques en français de l'oralité ou du récit informel, voire familier. Ainsi utilise-t-il régulièrement la dislocation, qui consiste à « détacher un constituant [...] en tête ou en fin de phrase 
et à le reprendre ou à l'annoncer par un pronom» (Ballard 2004: 254) et ce, dès l'incipit du roman (voir 2.2.3. pour le TD):

(25) Vous savez rien de moi si vous avez pas lu un livre qui s'appelle Les Aventures de Tom Sawyer, mais ça mange pas de pain. Ce livre, c'est Mr Mark Twain qui l'a fait, et il a dit la vérité vraie, en grande partie. Certaines choses, il les a exagérées, mais en grande partie il a dit la vérité. Ça fait rien. J'ai jamais connu quelqu'un qu'a pas menti une fois ou une autre, sauf tante Polly, ou la veuve, ou peut-être Mary. Tante Polly - elle, c'est la tante Polly de Tom - et Mary, et la veuve Douglas, on en parle dans ce livre, qui dans l'ensemble reflète la vérité; avec quelques exagérations, comme je l'ai dit tout à l'heure.

(Twain 1884/2008, traduit par Hoeffner: 11)

Même si l'on en trouve des exemples chez les autres traducteurs, c'est chez Hœpffner que les occurrences sont les plus nombreuses (voir par exemple Hœpffner 2008: 170).

On notera aussi qu'en ce qui a trait au rendu de l'oralité pour les phrases interrogatives, Hœpffner préserve l'ordre canonique avec l'interrogation marquée par l'intonation montante, ce qui n'est pas le cas chez les autres traducteurs:

(26a) I says to myself, if a body can get anything they pray for, why don't Deacon Winn get back the money he lost on pork?

(Twain 1884/1977: 14)

(26b) Je me dis, si les gens, ils peuvent obtenir tout ce qu'ils veulent en priant, pourquoi le diacre Winn, il a pas retrouvé l'argent qu'il avait perdu avec les porcs?

(Twain 1884/2008, traduit par Hopffner: 25)

Hœpffner parvient donc, par le recours aux diverses possibilités syntaxiques offertes par le français, à préserver le caractère oral et familier qui caractérise le langage de Huck.

\subsubsection{Grammaire}

Sur le plan grammatical, et plus particulièrement temporel, Hœpffner utilise le passé composé tout au long du roman, Jenn (2004a: 306) notant l'incompatibilité du passé simple avec le discours de Huck, temps néanmoins utilisé par les trois autres traducteurs, dans des proportions diverses. On remarque en outre chez Hœpffner le recours à diverses incorrections grammaticales, comme le conditionnel avec si, là où l'on trouve le conditionnel passé et le subjonctif imparfait chez Nétillard, les deux autres traducteurs ne rendant pas le passage:

(27a) I wished the widow knowed about it.

(Twain 1884/1977: 63)

(27b) J'aurais bien voulu que la Veuve le sût [...].

(Twain 1884/1993, traduit par Nétillard 1948: 94)

(27c) Si seulement la veuve l'aurait su.

(Twain 1884/2008, traduit par Hopffner: 118)

ou encore le que, subordonnant passe-partout, comme l'appelle Françoise Gadet (dans Muller 1996: 81):

(28a) "Well," I says, "s'pose we got some genies to help us - can't we lick the other crowd then?"

(Twain 1884/1977: 16-17) 
(28b) «Ah bon, que je dis, et si qu'on demandait à des génies de nous aider, nous - on pourrait pas aplatir les autres, alors?»

(Twain 1884/2008, traduit par Hopffner: 29)

On trouve également la locution malgré que, dont l'emploi est critiqué lorsqu'il est suivi du subjonctif, et a fortiori de l'indicatif:

(29a) The straps was broke off of it, but barring that, it was a good enough leg, though it was too long for me and not long enough for Jim [...].

(Twain 1884/1977: 45)

(29b) [Huck et Jim ont trouvé une jambe de bois.]

Les courroies étaient cassées mais, à part ça, c'était vraiment une belle jambe, malgré qu'elle était trop longue pour moi et trop courte pour Jim [...].

(Twain 1884/2008, traduit par Hopffner: 85)

ainsi que l'auxiliaire avoir avec certains verbes comme les verbes pronominaux qui régissent normalement l'utilisation de l'auxiliaire être:

(30a) I was pretty tired, and the first thing I knowed, I was asleep. When I woke up I didn't know where I was, for a minute.

(Twain 1884/1977: 32)

(30b) J'étais plutôt fatigué, et la première chose qui s'est passée, c'est que je me suis endormi. Quand je m'ai réveillé j'ai pas su où j'étais pendant une minute.

(Twain 1884/2008, traduit par Hopffner: 57)

Ce que cette étude sur corpus nous montre, c'est que Hopffner rompt avec les traducteurs précédents, par l'inventivité dont il fait preuve, par le recours tous azimuts aux diverses ressources de la langue, et par les écarts qu'il s'autorise à faire vis-à-vis du français standard, en introduisant délibérément des incorrections grammaticales, que l'on entend néanmoins chez les jeunes ou chez les locuteurs peu éduqués.

\section{Réception de la traduction de Bernard Hœpffner}

Après ce survol de quelques aspects de la traduction de Hœpffner, on peut se demander avec Antoine Berman (1995: 92) si «le traducteur a réalisé un véritable travail textuel, a fait texte, en correspondance plus ou moins étroite avec la textualité de l'original». Un des critères pour en juger peut consister à voir comment cette traduction a été reçue, même si, évidemment, on ne peut négliger le caractère biaisé des commentaires concernant un nouveau roman ou une nouvelle traduction, qui relèvent d'un style éditorial particulier et ont assez souvent tendance à faire dans l'hyperbole et le laudatif. Par ailleurs, les appréciations portent sur le roman dans son ensemble là où nous nous sommes focalisée sur la voix de Huck uniquement.

Nous avons constaté que la presse française, par l'intermédiaire de sites internet et de blogs littéraires, a tout d'abord bien perçu l'ouvrage comme une traduction à part entière, citant à chaque fois l'éditeur et/ou le nom du traducteur, ce qui n'est pas si courant en France:

«Tom Sawyer» et «Huckleberry Finn», des romans pour enfants? Une nouvelle traduction restitue à Mark Twain, qu'admirait tant Hemingway, sa place parmi les plus grands. C'est un choc. Des «Aventures de Tom Sawyer», on gardait le souvenir enchanté des lectures qui, pendant des heures, nourrissent les jeux de l'enfance. Dans la nouvelle 
traduction que publient aujourd'hui les éditions Tristram, on découvre l'énergie, la drôlerie et la vitalité d'un style brassant les dialectes des esclaves nègres, les grossièretés des voyous du Mississipi [sic], les fautes de syntaxe de gamins qui ne pensent qu'à sécher l'école. Et l'on mesure combien Mark Twain, dans son souci d'écrire comme on parle, est le contemporain du Dostoïevski chaotique révélé il y a quelques années par André Markowicz. Rien de plus spectaculaire, à cet égard, que les "Aventures de Huckleberry Finn», où un ado passablement illettré raconte, à la première personne, ses mois de vagabondage fluvial en compagnie d'un esclave marron. On y est à michemin entre Dickens, Lucky Luke et Raymond Queneau». (Leménager 2008)

Nombreux sont les critiques qui soulignent que le roman de Twain a trop longtemps été cantonné au rayon jeunesse en France, ce que la traduction de Hœpffner parviendra peut-être à changer :

Bernard Hœpffner a retraduit deux livres cultes de Mark Twain, que l'on considérait - à tort - comme des livres destinés aux plus jeunes: Les aventures de Tom Sawyer, et Les aventures de Huckleberry Finn. Nous pensions connaître Mark Twain, nous avions lu ces livres plus jeunes, nous avions également parfois suivi les aventures de Tom Sawyer sur le petit écran, en dessins animés. Et pourtant. Ces deux livres traduits par Bernard Hœepfner apparaissent à tous ceux qui les ont lus comme des immenses découvertes. (Anonyme 2008 ${ }^{24}$ )

Ce qui est surtout mis en avant, c'est le caractère novateur de la traduction de Hœpffner, qui se démarque de ses prédécesseurs:

Fini les adaptations édulcorées des «Aventures de Tom Sawyer» et surtout tronquées de "Huckleberry Finn », monument du roman américain selon Hemingway himself. Deux traductions rendent enfin hommage à Mark Twain, au génie novateur de la littérature anglo-saxonne. [...] C'est cette inventivité, cette inconvenance et cette vitalité, cette oralité savamment et puissamment recréée, que s'est attaché à restituer Bernard Hœpffner, dans une traduction délurée et pleine d'ardeur, où il n'hésite pas à inventer des néologismes, à bousculer la grammaire et la syntaxe de la langue française. (Crom 2008)

Tom Sawyer et Huckleberry Finn comme vous ne les avez jamais lus. Une nouvelle traduction redonne toute leur verve aux «kids» de Mark Twain. [...] Bernard Hœpffner vient de retraduire [Tom Sawyer et Huckleberry Finn] avec une faconde délicieusement rabelaisienne. [...] Traduites en France dès la fin du XIX ${ }^{\mathrm{e}}$ siècle, les odyssées déjantées - et parfois tranquillement immorales - de Mark Twain furent souvent édulcorées, trahies, repeintes au Ripolin de la bienséance. Grâce à Bernard Hœpffner, qui s'est déjà frotté à Joyce et à Melville, elles nous reviennent avec toute leur saveur originale, leur sulfureuse insolence, leurs mots tordus, leur flamboyante syntaxe qui s'embrase dans les fournaises du Deep South. [...] Il fallait donc que ça décoiffe pour que ce soit vraiment «autant tique», comme dit Huck en nous laissant «putréfiés d'étonnement » avant de décliner la longue liste de ses «misérabilités» [...]. (Clavel $2008^{25}$ )

Certes, Berman (1995: 66, note 61) nous a appris à nous méfier des impressions trompeuses et des qualificatifs employés par les critiques, mais force est de constater que les commentaires sont dithyrambiques, en particulier pour ce qui est du rendu des voix dans le roman de Twain, du travail stylistique, des nuances entre les idiolectes:

De ce point de vue, le travail de Bernard Hœpffner, qui a traduit le roman pour les éditions Tristram, force le respect par son inventivité linguistique et le respect qu'il témoigne à ces «nuances» évoquées par l'auteur. [...] Grâce au travail d'un éditeur attaché à la réception des œuvres majeures et conscient de leur étonnante modernité, 
les lecteurs francophones ont maintenant accès à ce roman flamboyant dans une traduction qui lui restitue toute sa force littéraire. (Meyrignac 200926)

C'est donc à un étonnant voyage à travers une littérature riche d'une langue et de personnages, illustrant la société sudiste du xıxème [sic] siècle, que nous invite Bernard Hœpffner. Il nous offre ici une traduction brillante, d'une vitalité et d'une drôlerie incontestables, dans un style brassant les dialectes des esclaves, les grossièretés des voyous du Mississippi, les fautes de syntaxe de gamins adeptes de l'école buissonnière. Plus encore qu'un voyage, cette croisière le long du Mississippi est un bain de jouvence, un enchantement de chaque instant à conseiller aux lecteurs de tous les âges. (MaliverPerrin 200927)

Nous avons vu en effet que Hœpffner n'avait pas ménagé sa peine pour essayer de rendre compte des spécificités langagières, littéraires et esthétiques de la voix de Huck, choisissant de laisser libre cours à une inventivité à laquelle les précédents traducteurs n'avaient pas eu recours, mais que d'aucuns diront débridée, dans la mesure où Hœpffner utilise des néologismes qui pourront paraître anachroniques par rapport au texte de Twain. Le traducteur avoue d'ailleurs dans une interview à propos des difficultés rencontrées: «D’abord, on peut toujours commettre des anachronismes involontaires, ou se tromper sur certains mots techniques» (Hœpffner, dans Leménager 2008). Il faut en outre signaler que Hœpffner indique avoir employé parfois sciemment des anachronismes. Ainsi, en réponse à la question de savoir si sa traduction de Twain est une traduction «pour adultes», Hœpffner répond:

Bernard Hœpffner. - [...] Ce serait excessif. J'ai fait exprès d'utiliser quelques petits trucs pour plaire aux enfants: comme certains anachronismes, que personne ne remarquera, mais que je me suis amusé à glisser... «Stylé» par exemple, pour traduire "styled». L'un des enfants avec qui je vis m'avait complimenté sur mes nouvelles baskets: "t'es stylé Bernard!». Je me suis dit que Huck pourrait très bien dire cela. Le problème, c'est que dans dix ans, ça paraîtra sans doute vieillot. (Leménager 2008)

Ceci est à l'opposé du projet de Jenn dans sa thèse, où les propositions de retraduction

sont toutes situées dans l'optique du rétablissement de la rhétorique enfantine et se veulent plausibles. La plausibilité implique de veiller à ce que ces propositions soient non anachroniques, c'est pourquoi certains termes sont accompagnés de leur date de première apparition telle qu'indiquée dans le Le Robert. (Jenn 2004a: 10 ; nous soulignons)

La question soulevée ici de l'anachronisme en traduction, avec deux avis contradictoires, ne saurait être traitée en quelques lignes et mériterait d'être poursuivie et approfondie ailleurs. Toutefois, on pressent qu'une étude minutieuse de la retraduction de Hœpffner dans son ensemble, se focalisant par exemple sur le sociolecte des autres personnages et sur le rendu des différences parfois subtiles entre les diverses voix du roman, pourrait mettre à jour quelques failles ou choix critiquables, qui pourraient nuancer les commentaires unanimement laudatifs des critiques.

\section{Conclusion}

L'étude sur corpus menée ici montre chez Hœpffner une prise en compte de la texture du roman de Twain, avec ses caractéristiques linguistiques, stylistiques et esthétiques qui en font un ouvrage si spécifique de la littérature américaine, un réel effort pour dépayser le lecteur et une conception bien arrêtée de ce que doit être la tâche du 
traducteur. Contrairement à certaines traductions précédentes, Hœpffner prend le parti de ne pas édulcorer le texte et fait preuve de la même inventivité que Twain, en recourant aux néologismes et aux mots-valises et en n'hésitant pas à malmener le français standard afin de rendre compte du caractère oral et familier qui caractérise la voix de l'adolescent qu'est Huck. Toutefois, malgré cette inventivité, la version de Hœpffner ne pourra jamais paraître aussi «novatrice», aussi moderne que l'a été le roman de Twain, car elle s'inscrit dans une temporalité différente: les moyens ici mis en œuvre par Hœpffner ne sont pas nouveaux, dans le sens où le "polysystème» français a déjà eu son Queneau, son Céline. La nouveauté réside peut-être davantage dans le fait d'avoir osé utiliser ces moyens pour la première fois dans une traduction de Huckleberry Finn, là où les traducteurs précédents restaient bien timides et où Queneau et Céline, écrivant directement en français, bénéficiaient de la position sacro-sainte d'auteur. Hœpffner revendique de livrer un texte qui soit perçu comme une traduction et de faire ressentir au lecteur qu'il lit «quelque chose qui n'est pas exactement du français, c'est-à-dire quelque chose qui n'a pas été directement écrit en français» (Hœpffner, dans Leménager 2008). On assiste ainsi à une entreprise de décentrement qui fait qu'on a effectivement l'impression en de nombreux endroits d'un texte «en français " plutôt que d'un texte «français», ce qui place cette traduction dans une sorte d'"entre-deux», le but avoué du traducteur étant de faire violence à la langue française. On peut se demander alors si cette retraduction réussit à «renouer avec un original recouvert par ses introductions, restituer sa signifiance, rassembler et épanouir la langue traduisante dans l'effort de restituer cette signifiance, lever, au moins en partie, cette défaillance de la traduction qui menace éternellement toute culture» (Berman 1990: 7). Il est bien entendu difficile de donner une réponse catégorique ici car nous nous sommes concentrée exclusivement sur Huck, et il resterait à étudier le rendu de la voix de Jim et des autres personnages afin de pouvoir émettre un jugement qui porte sur l'ensemble du roman traduit et de voir plus précisément quelle contribution cette version apporte dans le cycle des retraductions. On peut néanmoins avancer que cette nouvelle traduction propose un texte éminemment rajeuni qui, par son style, par les manipulations qu'il fait subir à la langue et le souffle qu'il apporte, devrait plaire aux (jeunes) lecteurs du $\mathrm{xxI}^{\mathrm{e}}$ siècle tout en leur offrant un dépaysement à la fois linguistique et culturel.

\section{NOTES}

* Membre de l'unité de recherche «Textes et Cultures», Équipe d'Accueil EA 4028.

1. Twain, Mark (Samuel Langhorne Clemens) (1884/1977): Adventures of Huckleberry Finn, An Authoritative Text, Backgrounds and Sources, Criticism. Sculley Bradley, Richmond Croom Beatty, E. Hudson Long et Thomas Cooley, dir. New York/London: Norton.

2. Twain, Mark (1884/2008): Aventures de Huckleberry Finn. (Traduit par Bernard HeppFner). Auch: Tristram. Hœpffner indique comment le projet de retraduction des Aventures de Tom Sawyer et des Aventures de Huckleberry Finn a vu le jour: "Aleksander Grudjijic a essayé de faire accepter le projet par Actes-Sud, sans succès; mais les éditions Tristram ont suivi immédiatement et avec le plus grand enthousiasme» (Monti 2008). Nathalie Crom donne les précisions suivantes concernant la maison d'édition: «Il se trouve que le projet du traducteur a rencontré, il y a deux ans et demi, le désir déjà attisé d'un éditeur: Tristram - la maison d'édition fondée et animée, à Auch, par Jean-Hubert Gailliot et Sylvie Martigny, et qui tire son nom du chef-d'œuvre de l'Anglais Lawrence Sterne, qu'ils ont fait traduire en 1999»(Crom 2008 - voir note 13).

Monti, François (2008) : Drôles de projets: quelques nouvelles de Bernard Hœpffner. Le Fric Frac Club, (3 mars 2008). Consultée le 6 novembre 2011, <http://fricfracclub.com/spip/spip.php?article 267>. 
3. Twain, Mark (1884/1993): Les aventures d'Huckleberry Finn. (Traduit par Suzanne NÉTILLARD, 1948). Paris: J’ai lu.

4. Twain, Mark (1884/1990): Les aventures d'Huckleberry Finn, l'ami de Tom Sawyer. (Traduit par André Bay, 1960). Préface d'André Bay. In: Mark Twain, Euvres. Introduction par Pierre Brodin. Paris: Laffont.

5. Twain, Mark (1884/1994): Les aventures de Huckleberry Finn. (Traduit par André BAy, 1960). Introduction, notes, bibliographie et chronologie par Claude GrImAL. Paris: GF-Flammarion.

6. Twain, Mark (1886): Les aventures de Huck Finn, l'ami de Tom Sawyer. (Traduit par William-L. Hughes). Illustrations par Achille Sirouy. Paris: Hennuyer. Version numérisée par l'Université de l'Illinois Urbana-Champaign. Consultée le 9 janvier 2009, <www.archive.org/stream/lesaventures deh00twai/lesaventuresdeh00twai_djvu.txt>.

7. Berman (1995: 76) donne la définition suivante du projet de traduction: «Le projet définit la manière dont, d'une part, le traducteur va accomplir la translation littéraire, d'autre part, assumer la traduction même, choisir un "mode" de traduction, une "manière de traduire"." Il ajoute: "[le critique] doit lire la traduction à partir de son projet, mais la vérité de ce projet ne nous est finalement accessible qu'à partir de la traduction. Car tout ce qu'un traducteur peut dire et écrire à propos de son projet n'a de réalité que dans la traduction» (Berman 1995: 77).

8. Voir Lavoie $(1994 ; 2002 \mathrm{a} ; 2002 \mathrm{~b})$, Gouanvic (2004) et Jenn (2004a; 2004b).

9. Brodin, Pierre (1948/1990): Introduction. In: Mark Twain (1884/1990): Les aventures d'Huckleberry Finn, l'ami de Tom Sawyer. (Traduit par André BAY, 1960). Paris: Laffont.

10. Grimal, Claude (1994): Introduction, notes, bibliographie et chronologie. In: Mark Twain (1884/1994): Les aventures de Huckleberry Finn. (Traduit par André BAY, 1960). Paris: GF-Flammarion. Cette citation ainsi que la suivante se trouvent dans l'introduction.

11. Coveney, Peter (1985): Introduction. In: Mark Twain (1884/1985): The Adventures of Huckleberry Finn. Harmondsworth: Penguin Books, 9-41.

12. BAY, André (1960/1990): Note sur la traduction. Les aventures d'Huckleberry Finn, l'ami de Tom Sawyer. (Traduit par André BAy, 1960). In: Mark Twain, Euvres. Paris: Laffont, 178.

13. Свом, Nathalie (2008): «Mark Twain» enfin en français. Télérama (21 septembre 2008). 3062. Consultée le 9 janvier 2009, <http://www.telerama.fr/livre/enfin-en-francais,33687.php>.

14. Audrerie, Sabine (2008): Mark Twain tel qu'on ne l'avait jamais lu. La Croix. (1 ${ }^{\text {er }}$ octobre 2008). Consultée le 6 novembre 2011, <http://www.la-croix.com/Culture-Loisirs/Culture/Livres/MarkTwain-tel-qu-on-ne-1-avait-jamais- lu-_NG_-2008-10-01-678338>.

15. Hœpffner fournit d'autres indications dans une entrevue réalisée par Grégoire Leménager en 2008 (voir note 17): «Certaines pages [de Huck Finn] sont d'ailleurs assez dures pour des enfants, et notamment celles, totalement inédites en français, qui figurent dans cette traduction : une sordide histoire de tonneau fantôme qui hante le Mississipi [sic]; le passage où Jim raconte s'être retrouvé, dans une salle de "dissexion", la tête entre les jambes d'un cadavre - c'est-à-dire le nez contre le sexe du cadavre. Le manuscrit a été retrouvé il y a quelques années: c'est typiquement le genre d'histoire que glissait Twain dans son récit pour choquer ses proches, au moment où il le leur lisait. C’est probablement sa femme qui se serait opposée à la publication de cet épisode-là.»

16. Hepffner, Bernard (2008): Note du traducteur. In: Mark Twain (1884/2008): Aventures de Huckleberry Finn. (Traduit par Bernard Hepffner). Auch: Tristram, p. 440.

17. Twain, Mark (1884/1985): The Adventures of Huckleberry Finn. Harmondsworth: Penguin Books.

18. Leménager, Grégoire (2008): C’est Mark Twain qu'il ressuscite. Le Nouvel Observateur. Bibliobs (18 septembre 2008). Consultée le 8 janvier 2009, <http://bibliobs.nouvelobs.com/romans/20080918. BIB2032/c-039-est-mark- twain-qu-039-il-ressuscite.html >.

19. Dictionnaire du Moyen Français (1330-1500), DMF1. Consultée le 6 novembre 2011, <http://atilf. atilf.fr/dmf.htm>.

20. Trésor de la langue française informatisé. Consultée le 12 novembre 2011, <http://atilf.atilf.fr/ dendien/scripts/tlfiv5/visusel.exe?12;s=3877145715;r=1;nat=;sol=1;>.

21. Robert, Paul (2004): Le Nouveau Petit Robert: Dictionnaire alphabétique et analogique de la langue française. Paris: Dictionnaires Le Robert.

22. Le terme attrimer figure dans Le nouveau dictionnaire complet du jargon de l'argot, Le langage des voleurs dévoilé, d'Arthur Halbert d'Angers. Consultée le 30 juin 2009, <http://www.gutenberg.org/ files/25949/25949-8.txt>.

23. Voir Queneau (1947: 64; 1959: 12, 14, 17, 19, 42, 104, 142) et Céline (1952: 10). CÉLINE, Louis-Ferdinand (1952): Voyage au bout de la nuit. Paris: Gallimard. Queneau, Raymond (1947): Exercices de style. Paris: Gallimard. Queneau, Raymond (1959): Zazie dans le métro. Paris: Gallimard. 
24. Anonyme (2008): Bernard Hopffner, derniers livres traduits. Le Bloc-notes Lekti. 30 septembre 2008. Consultée le 9 janvier 2009, <http://www.lekti-ecriture.com/bloc-notes/index.php/post/ 2008/09/30/Bernard-Hœpffner-derniers-livres-traduits>.

25. Clavel, André (2008): Les chenapans du Mississippi. L’Express, 4 décembre 2008. Consultée le 13 février 2009, <http://www.lexpress.fr/culture/livre/les-aventures-de-tom-sawyer-aventures-dehuckleberry-finn_823191.html>

26. Meyrignac, Mathieu (2009): Note de lecture sur Aventures de Huckleberry Finn. Centre national du livre. Consultée le 9 janvier 2009, <http://www.centrenationaldulivre.fr/?Aventures-dHuckleberry-Finn $>$.

27. Maliver-Perrin, Sandrine: Le chant du Mississippi. Montpellier: Librairie Sauramps. Consultée le 9 janvier 2009, <http://www.sauramps.com/article.php3?id_article $=4620>$.

\section{RÉFÉRENCES}

Ballard, Michel (2004): Versus: la version réfléchie. Des signes au texte. Vol. 2. Paris: Ophrys. Ben-ArI, Nitsa (1998): The Ambivalent Case of Repetitions in Literary Translations. Avoiding Repetitions: a "Universal" of Translation? Meta. 43(1):68-78.

Berman, Antoine (1990): La retraduction comme espace de la traduction. Palimpsestes. 4:1-7. Berman, Antoine (1995): Pour une critique des traductions: John Donne. Paris: Gallimard. Berman, Antoine (1985/1999): La Traduction et la lettre ou l'Auberge du lointain. Paris: Gallimard. Demanuelli, Claude (1993): Glissements progressifs vers... le texte d'oralisation. In: Michel Ballard, dir. La traduction à l'université, recherches et propositions didactiques. Lille: Presses Universitaires de Lille, 85-116.

Dupriez, Bernard (1984): Gradus, les procédés littéraires. Paris: Union Générale d'Éditions.

Gouanvic, Jean-Marc (1999): Sociologie de la traduction, la Science-Fiction américaine dans l'espace culturel français des années 1950. Arras: Artois Presses Université.

GouAnvic, Jean-Marc (2004): L'adaptation et la traduction: analyse sociologique comparée des Aventures de Huckleberry Finn de Mark Twain (1948-1960). Palimpsestes. 16:151-168.

Jenn, Ronald (2003): Transferring the Mississippi: Lexical, Literary and Cultural Aspects in Translations of Adventures of Huckleberry Finn. Revue française d'études américaines. 98:57-68.

Jenn, Ronald (2004a): La traduction de la rhétorique enfantine chez Mark Twain. Thèse de doctorat non publiée. Bordeaux: Université de Bordeaux.

Jenn, Ronald (2004b): From American frontier to European borders: the multiple bordercrossing of Twain's novels Tom Sawyer and Huckleberry Finn, résumé de l'intervention de l'auteur lors du congrès annuel de SHARP (Society for the History of Authorship, Reading and Publishing) à Lyon (20-23 juillet 2004), <http://ihl.enssib.fr/siteihl.php?page=172>, consultée le 13 janvier 2009.

Lavoie, Judith (1994): Problèmes de traduction du vernaculaire noir américain: le cas de The Adventures of Huckleberry Finn. TTR. 7(2):115-145.

Lavoie, Judith (2002a): Traduire pour aseptiser, Huck Finn revu et corrigé par W.-L. Hugues. Babel. 48(3):193-216.

Lavoie, Judith (2002b): Mark Twain et la parole noire. Montréal: Presses de l'Université de Montréal.

Maniez, Claire (1998): Les traductions françaises de The Adventures of Huckleberry Finn: production et réception. In: Annick DuperRay, dir. Discours et fictions d'Amérique: visions et révisions. Annales du monde anglophone. 7:71-84.

Muller, Marie Sylvine (1996) : Langue familière, parler populaire, particularisme régional dans Saturday Night and Sunday Morning d'Alan Sillitoe et sa traduction française. Palimpsestes. 10:49-75.

SAGOLs, Hélène (2005) : Raphäl Confiant: un langage entre attachement et liberté. Loxias. Loxias 9, mis en ligne le 15 juin 2005. Consultée le 23 mars 2009, <http://revel.unice.fr/loxias/ document.html?id=121>. 